\title{
A Novel Approach to Teaching Amplitude and Phase Distortion Concepts Using Time Domain Methods
}

\author{
Paul Crilly and Richard J. Hartnett \\ Department of Engineering, Electrical Engineering, \\ U.S. Coast Guard Academy, New London, CT \\ Paul.B.Crilly@uscga.edu
}

input and output signals on an oscilloscope, one can tell a lot about the amplifier. ${ }^{*}$ If the output and input only differ by a gain constant and perhaps a time shift, one can say that the amplifier does not suffer from amplitude or phase distortion. Conversely, if the output appears as a single sinusoidal wave, then the amplifier exhibits a great deal of amplitude distortion since it is acting as a filter. Thus by using wide-band periodic waveforms, one can determine the bandwidth of an amplifier, and reinforce student learning of Fourier series concepts from the time domain perspective. As a final example, consider a wide-band amplifier with large dynamic range and minimal amplitude distortion, implemented using negative feedback. One might expect that when driven by a square wave, the output waveform would also be square (except for scaling and delay). Instead because of phase distortion, the oscilloscope might display something very different from a square wave. Phase distortion in an otherwise high fidelity amplifier will cause the input harmonically related components to be delayed by different amounts, so the output will not match the input.

We find that the time domain techniques presented help students better appreciate the abstract concept of magnitude and especially phase distortion, help reinforce fundamental Fourier concepts, and can be accomplished without the added expense of network analyzers.

The following is a brief survey of how systems can be characterized with respect to distortion. Most of these are from the audio engineering literature with the focus on measuring distortion and not necessarily in explaining signal processing concepts. Griffiths [1] and Kuhn [2] describe the widely used method of square wave testing to measure amplitude distortion. They and most others who use this method do not deal with phase distortion, probably because the human ear does not readily pick up phase distortion. However, Lipschitz et al. [3] describes experiments on how the ear can perceive the effects of phase distortion. Cabot [4], [5] presents an excellent comprehensive treatment that describes the various types of distortion, and how these can be measured using single tone inputs and Fourier analysis. Blaess [6] and French

Most undergraduate laboratories have generators capable of generating waveforms that are rich in harmonics, as well as two channel oscilloscopes, where comparisons can be made between input and output signals. For example, if one drives an audio amplifier with a square wave and observes both the

* With many amplifiers, it is easy overdrive the amplifier and cause it to output a square wave. Therefore, it is mandatory that the amplifier is operated in its linear range. 
[7] use pulses and Fourier analysis to measure system distortion.

In this paper we first will re-state the definitions associated with distortionless systems, present a brief example of phase distortion and then present some simulations to illustrate what amplitude and phase distortions look like.

\section{Distortionless Systems}

A distortionless system is where the output signal has the same "shape" as the input except that the output is a scaled and delayed version of the input. More precisely, a system is distortionless if $y(t)=K x\left(t-t_{d}\right)$ with $x(t)$ and $y(t)$ the input and output, respectively, and $K$ and $t_{d}$ are constants. Thus the system function is $H(j \omega)=K e^{-j \omega t_{d}}$

where $|H(j \omega)|=K$ and $\angle H(j \omega)=-\omega t_{d}$

There are many types of distortion, but we will only consider amplitude and phase distortion.

Amplitude distortion is when the $K$ term is not constant or is not "flat" over all input frequencies. One example is a low pass filter.

Phase or delay distortion occurs when $\angle H(j \omega)$ is nonlinear with frequency. This often occurs in feedback systems.

Unlike amplitude distortion, phase distortion is not easily observed and does not have the same degree of coverage in beginning signals and systems courses. Therefore, we present a brief example of a system with and without phase distortion.

Consider a system with flat frequency response and linear phase with an input of

$x(t)=10 \cos \frac{\pi}{2}(t-2)+2 \cos \frac{\pi}{6}(t-2)$

and $H(j \omega)=5 e^{-j 3 \omega}$.

It can be shown that the output response is,

$y(t)=50 \cos \frac{\pi}{2}(t-5)+10 \cos \frac{\pi}{6}(t-5)$

We observe the response meets the non-distortion criteria as defined above.

We now consider the same input to a system with constant amplitude, but nonlinear phase with

$H(j \omega)=5 e^{-j \omega^{2}} \Rightarrow 5 \angle-\omega^{2}$.

It can be shown that the output response is,

$$
y(t)=50 \cos \frac{\pi}{2}(t-3.57)+10 \cos \frac{\pi}{6}(t-2.52)
$$

While there is no amplitude distortion, the uneven relative time delays indicate the system has phase distortion.
Finally, in systems without phase distortion, if the input is symmetrical, then the output would also be symmetrical.

\section{Simulations}

To illustrate the effects of amplitude and phase distoration, we simulate systems that contain both distoration types, apply a square wave rich in harmonics and observe the responses. Figure 1 shows the output from a system that has moderate amplitude distortion and linear phase. Figure 2 is the output from a system with very little amplitude distortion and linear phase. In comparing Figures 1 and 2, we observe the effects of ampltude distortion. The output from the system with moderate ampltude distorton deviates greatly from a square wave as noted by the high degree of low frequency ripple in the output, whereas the output from the system with little amplitude distoration is a relatively close fascimile of the original square wave input. The observed ripple frequency is approximately that of the highest harmonic in the system's passband. We also observe that the time domain signal, with added ideal delay, could become an even function of time. This symmetry typically imples that the system in question has a reasonably linear phase characteristic.

Figure 3 simulates a system with the same degree of bandlimiting as the one in Figure 2, except it has moderate degree of nonlinear phase, hence phase distortion is present at the output. Note the output contains the same harmonic components as the system of Figure 2, but the phase distortion has caused the positive and negative amplitudes to develop a nonzero slope. This is due to the uneveness of the output times for each of the frequency components. This is further shown in Figure 4 which simulates a system with a much higher degree of phase nonlinearity than that of the system of Figure 3. Figure 4 shows that one of the higher order harmonics is disproportionally delayed, confirming the point made in Eq. (3). Certainly, other types of nonlinear phases can be simulated, but the main point is that systems with nonlinear phase distort the output in a different way than systems with amplitude distortion. Specifically, we note that no amount of ideal delay can produce an even function for the signals in Figures 3 and 4 . Such asymmetry typically implies that the system in question has some type of nonlinear phase characteristic.

Note at first observation of Figures 3 and 4, one might draw the conclusion that the signal doesn't have nonlinear phase, but exhibits the classic "overshoot" problem. At closer examination, the spike in amplitude occurs before the input changes whereas overshoot occurs due to the system's response to a change in its input.

\section{Conclusions}

We have developed a method to test a system for amplitude and phase distortion using a square wave input. The simulations indicate that this method is a viable alternative to 
the standard, but relatively expensive method of using a sweep generator with a network analyzer. Furthermore, in this technique, Fourier series concepts are reinforced.

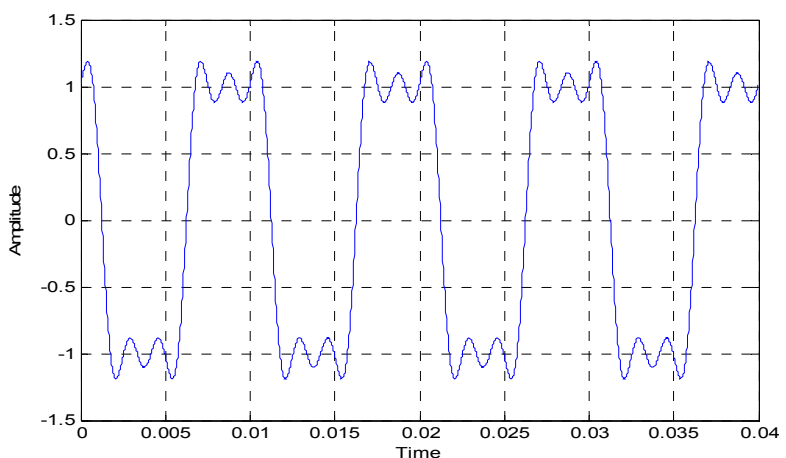

Figure 1. Waveform from system with large degree of amplitude distortion and linear phase. Ouptut only contains the the $1^{\text {st }}, 3^{\text {rd }}$ and $5^{\text {th }}$ harmonics. Although there is distortion, we note that with proper delay, the output could become an even function of time. This implies no phase distortion is present.

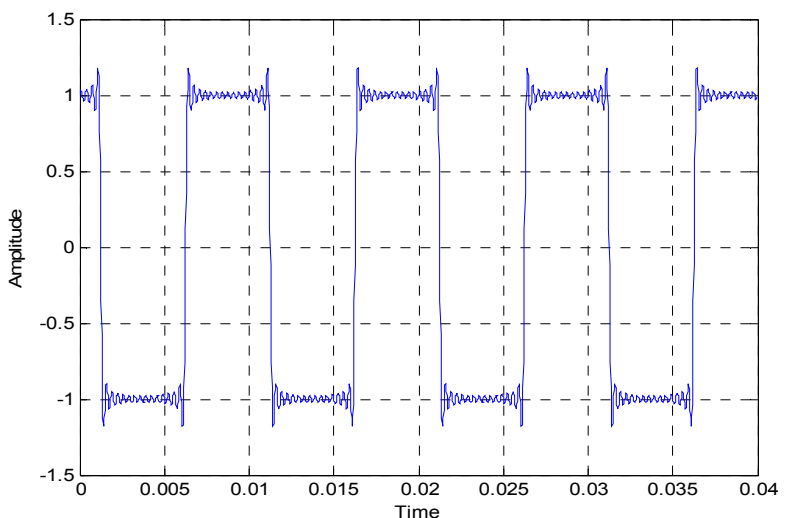

Figure 2. Waveform from system with negligible amplitude distortion and linear phase. Output contains 29 harmonics. Observe the time domain symmetry, such that an added delay could produce an even function of time. This implies the system must have exhibited linear phase.

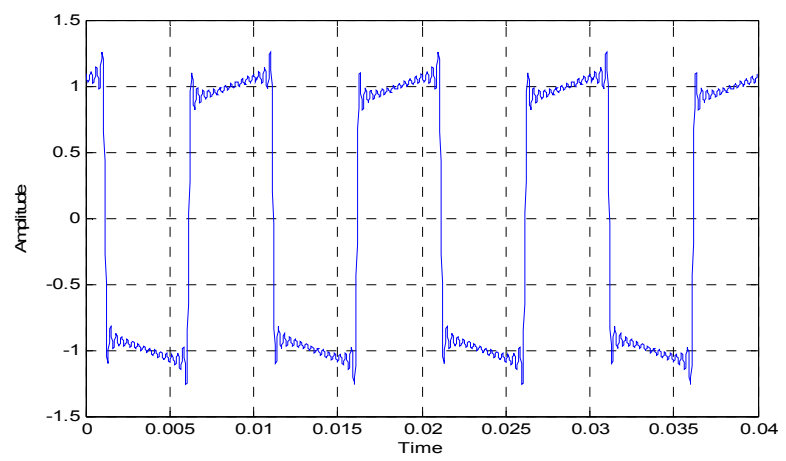

Figure 3. Waveform from system with negligible amplitude distortion and nonlinear phase. Output contains 29 harmonics. Unlike Figure 2, there is no ideal delay that could produce an even function, thus phase distortion must be present.

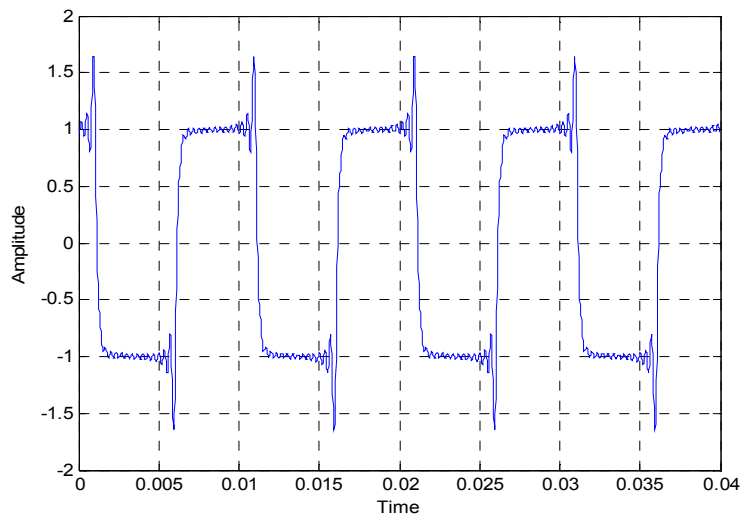

Figure 4. Waveform from system with negligible amplitude distortion and nonlinear phase. Output contains 29 harmonics. Again, there is no ideal delay that could produce an even function, so we know that phase distortion must be present.

\section{REFERENCES}

[1] G.F. Griffiths. (1968, June). Oscilloscope Testing of the Audio Amplifier [Online] Available: http://www.r-type.org/articles/art-125.htm

[2] K.A. Kuhn. (2008, Oct. 31). Square Wave Testing for Frequency Response of Amplifiers [Online]. Available: http://kennethkuhn.com/students/ee351/square_wave_testing.pdf

[3] S.P. Lipshitz et al. "On the Audibility of Midrange Phase Distortion in Audio Systems," JAES, vol. 30, No. 9, pp. 580-595, Sept. 1982.

[4] R.C. Cabot, "Fundamentals Of Modern Audio Measurement," JAES, vol.47, No. 9, 1999.Available:

http://www.electron.frba.utn.edu.ar/ jcecconi/Bibliografia/13\%20$\% 20$ Medicion\%20de\%20Amplificadores/Documentos/Fundamentals_M odern Audio Meas.pdf

[5] R. C. Cabot, "Method and Apparatus For Fast Response and Distortion Measurement," U.S. Patent 5,420,516, May 30, 1995.

[6] G. Blaess, "Method and Apparatus For Measurement of Attenuation and Distortion by A Test Object," U.S. Patent 4,275,446, June 23, 1981.

[7] R. C. French, "Transfer Function Measurement Using Fast Pulses," Electronic Engineering, pp. 516-519, Aug 1966 